Acta Crystallographica Section D

\section{Biological Crystallography}

ISSN 0907-4449

\section{Vulovic, ${ }^{a, b}$ B. Rieger,} L. J. van Vliet, ${ }^{b}$ A. J. Koster ${ }^{a}$ and R. B. G. Ravelli ${ }^{\mathbf{a} *}$

asection Electron Microscopy, Department of Molecular Cell Biology, Leiden University Medical Center (LUMC), PO Box 9600, 2300 RC Leiden, The Netherlands, and ${ }^{\mathbf{b}}$ Quantitative Imaging Group, Department of Imaging Science and Technology, Faculty of Applied Sciences, Delft University of Technology, Delft, The Netherlands

Correspondence e-mail: ravelli@lumc.nl

\title{
A toolkit for the characterization of CCD cameras for transmission electron microscopy
}

Charge-coupled devices (CCD) are nowadays commonly utilized in transmission electron microscopy (TEM) for applications in life sciences. Direct access to digitized images has revolutionized the use of electron microscopy, sparking developments such as automated collection of tomographic data, focal series, random conical tilt pairs and ultralarge single-particle data sets. Nevertheless, for ultrahigh-resolution work photographic plates are often still preferred. In the ideal case, the quality of the recorded image of a vitrified biological sample would solely be determined by the counting statistics of the limited electron dose the sample can withstand before beam-induced alterations dominate. Unfortunately, the image is degraded by the non-ideal point-spread function of the detector, as a result of a scintillator coupled by fibre optics to a $\mathrm{CCD}$, and the addition of several inherent noise components. Different detector manufacturers provide different types of figures of merit when advertising the quality of their detector. It is hard for most laboratories to verify whether all of the anticipated specifications are met. In this report, a set of algorithms is presented to characterize on-axis slow-scan large-area CCD-based TEM detectors. These tools have been added to a publicly available image-processing toolbox for $M A T L A B$. Three in-house CCD cameras were carefully characterized, yielding, among others, statistics for hot and bad pixels, the modulation transfer function, the conversion factor, the effective gain and the detective quantum efficiency. These statistics will aid data-collection strategy programs and provide prior information for quantitative imaging. The relative performance of the characterized detectors is discussed and a comparison is made with similar detectors that are used in the field of X-ray crystallography.

\section{Introduction}

Charge-coupled devices (CCDs) are used in nearly every scientific domain of life-science imaging, e.g. for transmission and fluorescence microscopy, optical and UV spectroscopy, digital photography, X-ray diffraction and imaging, and electron microscopy. Large-area CCD-based systems are the most common detectors on modern synchrotron beamlines (Ponchut, 2006) and are complemented by multiwire gas-filled chambers and novel photon-counting pixel arrays. The instant image access in electronic form, high sensitivity, low noise and versatile coverage from submicrometric to millimetric spatial resolution as well as the high reliability of commercial CCD cameras make them ideal for a wide range of applications. In transmission electron microscopy (TEM), however, there has been a considerable delay in adaptation to CCD technologies.
Received 25 May 2009

Accepted 6 August 2009 
In 1982, Roberts and coworkers reported the use of an array of $100 \times 100$ photosensitive elements to detect $20-100 \mathrm{keV}$ electrons directly (Roberts et al., 1982). The system demonstrated an excellent linearity between input and output signal and a high intrinsic gain, but had a limited spatial resolution compared with photographic film and suffered from radiation damage. They suggested initial conversion of the electron image to its photon counterpart, followed by detection of the latter by a CCD. Spence \& Zuo (1988) reported the use of such an indirect detection scheme, involving an electron scintillator, an optical coupler and a $576 \times 382$ pixel sensor. Many more experimental and commercial systems have since been reported (see references in Fan \& Ellisman, 2000). Direct access to digital data has enabled developments such as autotuning of the microscope (Krivanek \& Mooney, 1993), automated electron tomography (Koster et al., 1992), protein electron crystallography (Brink \& Chiu, 1994) and automated cryo-electron single-particle micrograph collection (Carragher et al., 2000).

Despite the many advantages of CCDs, some areas remain in which the applications of CCDs have been limited by certain characteristics that are inherent to CCD-based detectors (Downing \& Hendrickson, 1999). For example, for highresolution single-particle work film is still significantly better (Sander et al., 2005) than fibre-optic coupled CCD detectors; without binning of the CCD camera and at a magnification of $70000 \times$, film is better beyond $21 \AA$ resolution. For fourfold binning of the CCD camera and at very high magnification $(>300000 \times)$, film is reported to be superior beyond $7 \AA$ resolution. This might have contributed to the slow transition from film recording to digital imaging in the field of TEM. Until recently, large-area CCD cameras were only offered as third party add-ons to new TEMs. The relatively slow pace of adoption partially reflects the satisfactory performance of film recordings in terms of resolution and number of pixels after digitization, although both gaps are being closed. Commercial digital cameras are now available that have a larger image area than film (http://www.tvips.com/Prod_TF816.php). Detector systems based on newly developed CMOS hybrid-pixel technology which operate in noiseless single-photon counting mode are already commercially available for X-ray imaging and diffraction applications (http://www.dectris.com/). Hybrid pixel detectors are being developed for TEM applications (McMullan et al., 2007; Faruqi et al., 2003) and offer considerable scope for better characteristics compared with phosphor/fibre-optic coupled CCDs (Faruqi \& Henderson, 2007).

The incremental improvements in CCD technology, number of pixels, quality of phosphors/scintillators, fibre-optic coupling and electronics as well as emerging novel pixel-array detector technology will not make it easier for the user to select the right detector for an experiment from this heterogeneous landscape. Whereas well funded large user facilities might be able to keep up to date with the latest detector technologies, most academic laboratories will have to select a particular detector and use it for at least a decade. Even among a given category of detectors such as CCD cameras, the wide range of inconsistent, sometimes incomprehensible and often incomplete commercial specifications hamper the selection process. In this paper, we present a set of algorithms to characterize CCD detectors, which have been implemented in DIPlib, a publicly available software toolbox (http:// www.diplib.org) for MATLAB (The MathWorks Inc.). This should facilitate the commission of new detectors and help in the design of better data-collection strategies using existing detectors. A number of detector characteristics are recapitulated, such as readout noise, conversion factor, effective gain, point-spread function, modulation transfer function and detective quantum efficiency. Three of our own $4 \mathrm{k} \times 4 \mathrm{k}$ TEM imaging CCD detectors have been characterized. Only the user can judge whether a detector meets the needs of an experiment and the outcome depends on many other elements as well, including the electron source, optics and, above all, the sample. Therefore, the differences found for the three detectors are not judged upon and no reference is made to their manufacturers.

\section{Detector characterization}

To characterize a CCD detector and subsequently identify and correct artefacts, one needs to determine the contributions of all noise components, the effective gain, conversion factor, linearity of response, modulation transfer function (MTF) and detective quantum efficiency (DQE). Temporal noise randomly changes from frame to frame. It includes stochastic contributions such as dark-current noise, readout noise, photon noise, beam flicker, burst noise and shutter noise. There is also a source of fixed-pattern noise, especially in fibreoptic coupled digital cameras. This spatial noise does not vary from frame to frame and is caused by spatial variation in the thickness of the scintillator, fibre-optic coupling ('chicken wire' or broken fibres), dust, CCD bias pattern (in particular if multiple readout ports or composite CCDs are used) and other artefacts that produce variations in the pixel-to-pixel sensitivity and/or distortions in the optical path to the CCD or in the CCD chip itself. Flat-field correction is used to suppress fixed-pattern noise.

A corrected image $I_{\text {corr }}(x, y)$ can be obtained via (Aikens et al., 1989)

$$
I_{\text {corr }}(x, y)=\frac{I_{\text {raw }}(x, y)-\overline{I_{\mathrm{bg}}}(x, y)}{I_{\text {gain }}(x, y)},
$$

where $I_{\mathrm{raw}}(x, y)$ is the original uncorrected image, $\overline{I_{\mathrm{bg}}}$ is the average background image (see below) and $I_{\text {gain }}(x, y)$ is the image with normalized gain values for each pixel. In X-ray crystallography, a fibre-optic taper or lens system makes the conversion from raw images to corrected images more cumbersome, since the distortion of the demagnifying system needs to be accounted for. Furthermore, it is nontrivial to obtain the stable large uniform X-ray beam that is needed for collection of the data from which $I_{\text {gain }}(x, y)$ is obtained. Therefore, most X-ray detector manufacturers deliver their camera with tables for distortion and flat-field correction and the user only has to collect background images for the desired 
exposure time. The manufacturers' gain and distortion calibration normally remains adequate for a number of years. This also holds for X-ray detectors in which fibre-optic plates (1:1 magnification) rather than tapers are used.

Electron-microscopy detectors typically employ fibre-optic plates in combination with large sensor chips. Two popular large-area CCD sensors are the Fairchild CCD 485 and 486 (Eagle 4k, Gatan 4k, Tvips 4k). These sensors are also used for a number of X-ray detectors (Bruker APEXI and II, platinum 135/200/200C, Rayonix 135 and 165). No distortion corrections are required when these sensors are bonded to a fibre-optic plate. The electron microscopist can perform the background and gain calibration in a straightforward manner, as large uniform flat-field electron-beam illumination conditions are readily obtained with modern electron microscopes. Academic and commercial electron-microscope data-collection packages, such as Tia (http://www.fei.com/products/types/ fei-software.aspx), SerialEM (Mastronarde, 2005), UCSF software (Zheng et al., 2007), Leginon (Suloway et al., 2005) and Digital Micrograph (http://www.gatan.com/products/ software/), provide functionality for this camera-calibration step. The rate of recurrence at which background and gain calibration is required is significantly higher compared with $\mathrm{X}$-ray detectors and can vary from once a month to a few times a day depending on the camera manufacturer.

Correction of raw images does not require the same illumination conditions during acquisition of raw images and the white reference images. The optical density of a semi-thin scattering-contrast-dominated TEM sample can be modelled via the Lambert-Beer law,

$$
\log \left(\frac{I_{\text {sample }}}{I_{0}}\right)=-\alpha l,
$$

where $I_{0}$ is the incoming intensity, $I_{\text {sample }}$ is the outgoing intensity, $\alpha$ is the absorption coefficient and $l$ is the path length. In this equation, $I_{0}$ does not have to be a uniform beam. A near-uniform beam can be referred to as flood field (Moy et al., 1996). The explicit measurement of $I_{\text {sample }}$ and $I_{0}$ in electron microscopy is, for example, carried out in the Leginon package for the automatic characterization of the thickness of vitreous ice specimens (Carragher et al., 2000). In principle, the separate measurements of the gain-normalized image $I_{\text {gain }}$ in (1) and a flood-field image $I_{0}$ in (2) could be combined into one measurement. However, such a characterization would only remain valid as long as $I_{0}$ does not change. The flood-field image $I_{0}$ will change for different electron-beam settings, whereas the gain-normalized image $I_{\text {gain }}$ is independent of the electron optics and only alters with factors such as temperature.

To estimate the properties of a fibre-coupled CCD correctly, it is important to suppress statistical outliers ('zingers'; named after Zinger, 1961) in the reference images. These can be detected by measuring a large number of images under identical conditions. Cosmic rays and muons in particular can produce a burst of photons in the scintillator, leading to white spots or streaks in the image. Radioactive elements (essentially thorium) present in the fibre-optic tapers can also lead to zingers (Bourgeois et al., 1994). Other possible sources of zingers are $\mathrm{X}$-rays and burst noise ('popcorn noise'), the latter referring to a variety of electronic effects that can yield both increased and decreased pixel values.

The average background image $\overline{I_{\mathrm{bg}}}(x, y)$ will be different for different integration times. It has a time-independent offset, the average bias $I_{\text {bias }}(x, y)$, plus a time-dependent contribution from the spontaneous thermally induced generation of electron-hole pairs within the CCD, which is referred to as dark current. For typical exposure times in bright-field TEM imaging of biological samples ( $0.1 \mathrm{~s}$ to a few seconds), a linear relation may be assumed,

$$
\overline{I_{\mathrm{bg}}}(x, y)=I_{\text {bias }}(x, y)+t_{\exp } I_{\mathrm{dc}}(x, y),
$$

where $(x, y)$ denotes the pixel position, $t_{\exp }$ the exposure time of the CCD camera (or integration for the dark images) and $I_{\mathrm{dc}}(x, y)$ the average dark current in counts per second. The readout noise $I_{\mathrm{rn}}(x, y)$ is the standard deviation of a large series of background images $I_{\mathrm{bg}}$ measured at an exposure time at or near $0 \mathrm{~s}$,

$$
I_{\mathrm{rn}}(x, y)=\left\{\frac{1}{N} \sum_{i=1}^{N}\left[I_{\mathrm{bg}, i}(x, y)-I_{\text {bias }}(x, y)\right]^{2}\right\}^{1 / 2} .
$$

A flat-field (uniform) illumination of the camera will not result in a uniform response of the CCD, as each of the conversion steps from high-energy electrons to photo-induced electrons read from the CCD will introduce local amplification or attenuation of the signal. The scintillator will have variations in thickness; some parts could be blocked by artefacts such as dust, the coupling of the scintillator to the fibre-optic plate will have imperfections, the fibre-optic plate itself will leave a very strong pattern of individual fibres and fibre bundles, the coupling of the fibre-optic plate to the CCD will lead to location-dependent signal loss and the CCD itself has a nonuniform response. The combined effects are corrected for by means of a flat-fielding, which relies on the measurement of white reference (uniformly illuminated) images $I_{\text {white }}$ at one or multiple exposure times,

$$
I_{\text {gain }}(x, y)=\frac{\overline{I_{\text {white }}}(x, y)-\overline{I_{\mathrm{bg}}}(x, y)}{\left\langle\overline{I_{\text {white }}}-\overline{I_{\mathrm{bg}}}\right\rangle_{x, y}},
$$

where $\overline{I_{\mathrm{bg}}}$ is an average background image as calculated with (3) and $\bar{I}_{\text {white }}$ is an average white reference image calculated in a similar way. The notation \langle\rangle$_{x, y}$ is used to denote spatial averaging over the entire image.

The modulation transfer function (MTF) is a measure of how the signal amplitude is transferred for different spatial frequencies. It is calculated from the modulus of the Fourier transform of the point-spread function (PSF) of the detector. There are two common methods for experimental determination of the MTF, referred to as the noise and the edge method. The noise method is a stochastic method in which the camera is exposed to uniform illumination. The incoming signal may be considered as white noise that has a constant power spectrum over all spatial frequencies. The assumption is that this constant spectrum will be attenuated by the MTF of 
the camera as any other signal. The detector PSF is expected to be dominated by the fibre-optic plate scintillator and, therefore, to be isotropic. The absolute value of the Fourier transform of a uniformly illuminated image, angularly averaged, yields an overoptimistic MTF (Fan \& Ellisman, 2000). Angular averaging of the Fourier transform can be performed by creating rings in an image with a Gaussian profile $G(r, \sigma)$. The Gaussian-weighted sum of the modulus of the Fourier transform of the white-noise image $|F(u, v)|$,

$$
|F(r)|=\frac{\sum_{u, v} G(r, \sigma)|F(u, v)|}{\sum_{u, v} G(r, \sigma)},
$$

will yield the MTF after normalization. The edge method is a deterministic method and uses a uniformly illuminated straight sharp metal knife-edge which blocks the incident electrons on one side (Dainty \& Shaw, 1974). The knife-edge profile can be represented by a step function. An image of the knife-edge is taken with uniform illumination and is subjected to flat-field correction. The mean intensities on the dark and bright sides are calculated and used to normalize the image. An average edge profile from the slanted edge is extracted from the image. Differentiation of the one-dimensional edgespread function (ESF) gives the point-spread function (PSF) and, after Fourier transform and taking the modulus, a onedimensional cross-section of the detector's two-dimensional modulation transfer function. Assuming an isotropic MTF, an edge measurement in a single direction suffices.

Attenuation from the MTF alone would not spoil the image quality. If the signal is transferred up to Nyquist frequency and the MTF is known, one can, in theory, restore the image by deconvolution. In practice, deconvolution will be hampered by noise. The detective quantum efficiency (DQE) describes the noise added by the detector.

The DQE is defined as the squared ratio of the SNR between output and input signal

$$
\mathrm{DQE}=\left(\frac{\mathrm{SNR}_{\mathrm{out}}}{\mathrm{SNR}_{\mathrm{in}}}\right)^{2} .
$$

The noise of a stochastic scattering process is not transferred in the same manner as the signal (Rabbani et al., 1987). An electron is scattered in the scintillator and produces photons along its trajectory. These photons are scattered again. The process in the scintillator is therefore a complicated combination of scattering and amplification: the noise in the detected (output) signal is not simply the noise in the input signal attenuated by the MTF (Meyer et al., 2000). The signal and noise transfer differently as a function of spatial frequency; thus, the DQE becomes

$$
\operatorname{DQE}(u, v)=\frac{S_{\text {out }}(u, v)^{2} / \operatorname{NPS}_{\text {out }}(u, v)}{S_{\text {in }}(u, v)^{2} / \operatorname{NPS}_{\text {in }}(u, v)},
$$

where NPS refers to the noise power spectrum. In order to measure DQE the frequency dependence of the signal for a white image is approximated by

$$
S_{\text {out }}(u, v)=\overline{S_{\text {out }}} \operatorname{MTF}(u, v),
$$

where $\overline{S_{\text {out }}}$ is the mean of the signal $S_{\text {out }}$. Since the input signal is a Poisson process with constant expected value across the image, the expected variance and the expected mean of the signal are the same and are frequency-independent, i.e. $\operatorname{NPS}_{\text {in }}(u, v)=S_{\text {in }}(u, v)=\overline{S_{\text {in }}}$. The mean of the incoming signal equals the dose, $\overline{S_{\text {in }}}=N$. The conversion factor is given as $C=\overline{S_{\text {out }}} / \overline{S_{\text {in }}}$. The DQE can now be rewritten as

$$
\operatorname{DQE}(u, v)=C^{2} N \frac{\operatorname{MTF}^{2}(u, v)}{\operatorname{NPS}_{\text {out }}(u, v)},
$$

with

$$
\mathrm{NPS}_{\text {out }}=\mathrm{FT}\left[\sigma_{\text {shot }}(x, y)\right]^{2}+\mathrm{FT}\left[I_{\mathrm{rn}}(x, y)\right]^{2},
$$

where FT[] denotes a Fourier transform, $\sigma_{\text {shot }}(x, y)$ is the standard deviation per pixel owing to Poisson noise and $I_{\mathrm{rn}}$ is the readout noise. The noise from dark current is usually dominated by the readout noise and will be neglected in this analysis. The relative contribution of the readout noise to $\mathrm{NPS}_{\text {out }}$ is larger for lower dose and higher frequencies. (10) can also be expressed as

$$
\operatorname{DQE}(u, v)=\frac{\operatorname{MTF}^{2}(u, v)}{\operatorname{NNPS}(u, v)},
$$

where NNPS is the normalized noise power spectrum,

$$
\text { NNPS }=\frac{\text { NPS }_{\text {out }}}{C^{2} N}
$$

\section{Measurement methods}

Three of our in-house on-axis bottom-mounted cameras were characterized. These detectors, named $X, Y$ and $Z$, were mounted on Tecnai microscopes (FEI Company, The Netherlands) which were operated at $120 \mathrm{kV}$ voltage. Two of the microscopes have a lanthanum hexaboride $\left(\mathrm{LaB}_{6}\right)$ tip as cathode and the third one has a field emission gun (FEG). Each of the three CCD sensors has an active surface of $61.2 \times 61.2 \mathrm{~mm}, 4096 \times 4096$ pixels, a pixel pitch of $15 \mu \mathrm{m}$ and a $100 \%$ fill factor (http://www.fairchildimaging.com/). The cameras differ in the phosphor scintillator and fibre-optic plate that is coupled to the CCD sensor. The unbinned images were read out at $1 \mathrm{MHz}$ by four parallel readout ports employing 16 bit $\mathrm{AD}$ converters; at the maximum speed one can obtain 7.5 unbinned images per minute. The square images are framed by five (detectors $X$ and $Y$ ) and ten (detector $Z$ ) reference pixels in each direction; this frame should be excluded from the final image. All cameras were Peltiercooled to a set temperature of $248 \mathrm{~K}$ in order to decrease dark current. Image processing was performed using MATLAB (Mathworks) and the DIPimage toolbox (TU Delft, The Netherlands; http://www.diplib.org). Data were collected using $M A T L A B$ scripts inspired by the TOM toolbox (Nickell et al., 2005) and employing the TEMScripting Active $X$ server v.3.1.2 (Tecnai; http://www.fei.com/products/types/fei-software.aspx). 
All functions for camera characterizations can be found online at http://www.diplib.org/home22266.

\subsection{Removal of outliers}

Dark reference images were acquired with the column valves closed, i.e. there was no beam. A series of at least ten images were acquired under identical conditions (with the same integration time). Pixels with intensity fluctuations larger than ten times the standard deviation of the intensity of a pixel within the series were marked as outliers. Occasionally, the iterative procedure identified two outliers within a series of ten. Outliers from the white reference images were removed in a similar way.

\subsection{Bias, dark current and readout noise}

After outlier removal, the bias and dark current was determined for every pixel by analyzing a total of 100 dark reference images measured at ten different exposure times. The range of exposure times was $0.05-10 \mathrm{~s}$. The dark current was determined for each pixel from the slope of a linear leastsquares fit of the dark images versus exposure time. The offset of this fit gave the bias.

The ten dark reference images with the smallest exposure time were used to calculate the readout noise by computing the standard deviation per pixel within the series.

\subsection{Detector effective gain measurements}

Two different approaches were used to determine the effective gain of each detector, one based on white reference images and the other based on gradient images.

White reference ( $\left.I_{\text {white }}\right)$ images were acquired with different exposure times using a constant uniform illumination of the detector. The beam was spread to be wider than the diameter of the fluorescent screen (165 mm) and it was slightly (15 mm) shifted from the optical axis in random directions between the acquisition of successive images in order to average out any potential non-uniformities in the illumination. The average background $\overline{I_{\mathrm{bg}}}$ was subtracted from these images. Outliers were removed as described above. Series of at least ten repeated exposures were made for seven different exposure times (range 0.05-2.5 s). Apart from the beam shift and exposure time, the illumination conditions were kept constant during the acquisition of all images. The spatial median of the intensities of all pixels within each quadrant was determined for each exposure time. Pixels with an average intensity that differed by more than $1 \%$ from this median were excluded from subsequent calculations. The variance of the pixel intensity within the series was determined for each selected pixel. The mean of the variance $\langle\operatorname{var}(I)\rangle_{x, y}$ and the mean of intensities $\langle I\rangle_{x, y}$ over the selected pixels in each quadrant were computed. A plot of the mean variance versus the mean intensity was made with dots representing the pairs $\left[\langle I\rangle_{x, y},\langle\operatorname{var}(I)\rangle_{x, y}\right]$ for each exposure time. The slope of a linear least-squares fit of this plot gave the effective gain of the camera.
The effective gain was also determined using gradient images (Vliet et al., 1998). A series of at least ten repeated measurements were made of a highly non-uniform beam. For the $\mathrm{LaB}_{6}$ microscopes, an intensity gradient was achieved by imaging the blurred beam edge at very high magnification. Since blurring of the beam edge is difficult to achieve for a FEG source, astigmatism of the condenser lens was used. As in the previously decribed method, outliers were removed, the average background $\overline{I_{\mathrm{bg}}}$ was subtracted, and the mask determined above was applied. The intensities in the gradient images were distributed into 100 bins. The variance and the mean of the intensity were calculated for each bin. The effective gain of the camera was again determined as described above.

\subsection{Bias correction, gain normalization and pixel response}

The white reference images described above were also used to check the linearity of the pixel response. Similar to the calculation of $\overline{I_{\mathrm{bg}}}$ (3), a linear least-squares fit of intensity versus exposure time was computed for each pixel to yield the average white image $\overline{I_{\text {white }}}(x, y)=I_{\text {bg }}(x, y)+t_{\exp } I_{\text {slope }}(x, y)$. The linearity of the pixel response as a function of the exposure time was checked by computing $R^{2}$, the square of the sample correlation coefficient between the measured and predicted values. The gain-normalization image $I_{\text {gain }}(x, y)$ was calculated using $\overline{I_{\text {bg }}}$ and $\overline{I_{\text {white }}}(x, y)(5)$.

Prior to the correction of a raw image using (1), border pixels had to be excluded from analysis. This border was five pixels wide for detectors $X$ and $Y$ and ten pixels wide for detector $Z$.

\subsection{Modulation transfer function (MTF)}

Ten flat-field corrected uniformly illuminated images taken with $1 \mathrm{~s}$ exposure time were used to calculate the MTF via the noise method. In order to avoid problems arising from averaging too few data points at low spatial frequencies, we used a variable standard deviation for the Gaussian in (6), namely $\sigma=2.5$ at low frequencies and $\sigma=0.9$ at higher frequencies. Note that the angular averaging takes place in the reciprocal domain and that $\sigma$ is expressed in number of bins. Individual MTF curves were calculated for each of the ten images; the final noise-method MTF was an average of these.

Both the beam stop and the diffraction aperture were used to determine the MTF via the edge method. The beam stop was placed directly above the fluorescent screen at a slightly inclined angle with respect to the pixel array (Samei et al., 1998). Ten images of the edge were taken with uniform illumination and subjected to outlier rejection and flat-field correction. The mean intensities on the bright and dark sides of the beam stop were calculated and used to normalize the image. The average edge profiles from the slanted beam-stop edge were extracted from the image. The edge profiles were oversampled by a factor of eight and processed according to Mullikin et al. (1994). Averaging of 128 lines along the edge suppressed the noise and yielded a one-dimensional edgespread function (ESF). The point-spread function (PSF) of the 


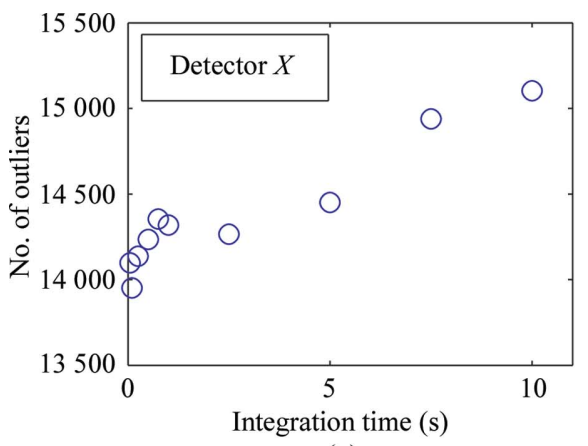

(a)

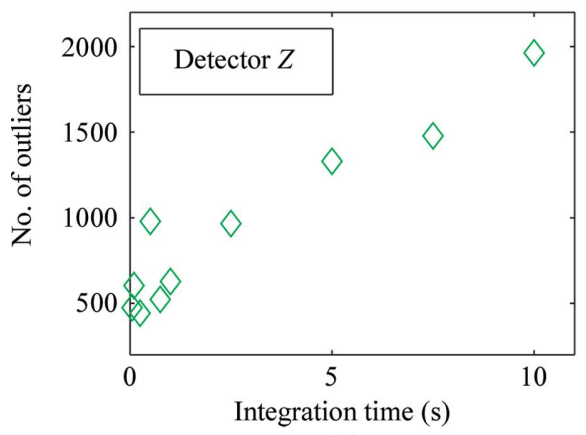

(c)

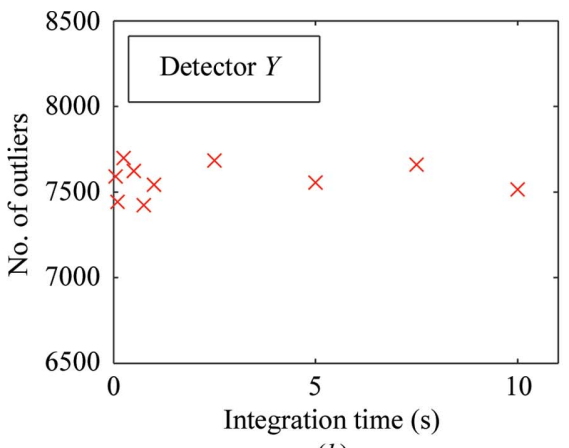

(b)

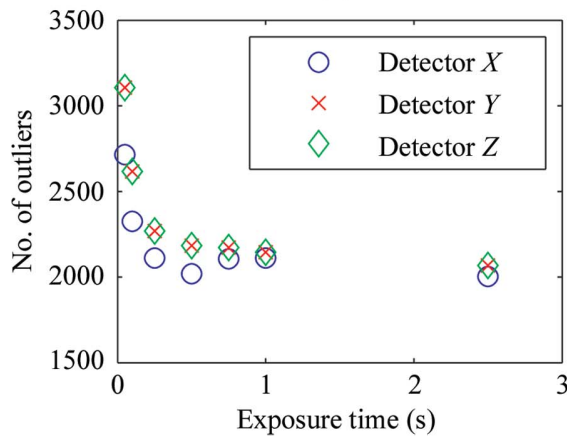

(d)

Figure 1

Number of outliers versus time. $(a)-(c)$ show the number of outliers in the dark images and $(d)$ shows those in the white reference images. Detectors $X$ and $Z$ show an increase in the number of outliers with integration time in the dark references. The number of outliers in the white references was comparable for the three detectors
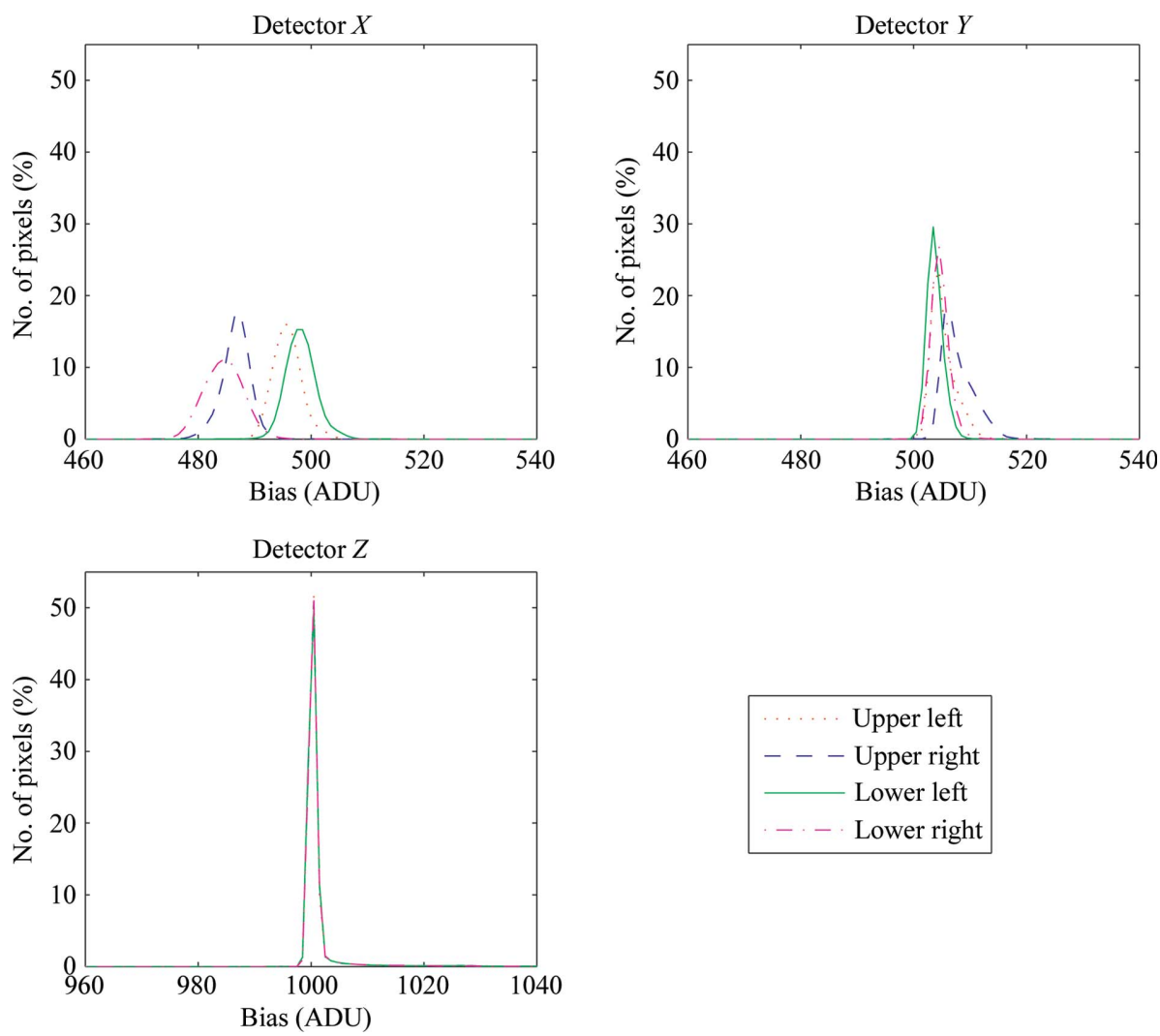

Figure 2

Histogram of the bias for each quadrant of the three detectors. The bin width of the histogram is 1 ADU. The average bias is 492, 506 and 1002 ADUs for detectors $X, Y$ and $Z$, respectively. Detector $Z$ shows the smallest spread of the bias.

detector was obtained by computing the derivative of the ESF via finite difference. Owing to Poisson statistics, it proved to be necessary to reduce the noise of the bright side of the edge by setting the tails of the PSF to zero. Individual MTFs were obtained after down-sampling the PSF to the original pixel pitch and computing the magnitude of the Fourier transform. We repeated this procedure for ten images and averaged the ten MTFs to obtain a more robust estimation.

Images of the diffraction aperture were taken at low magnification of the projection lens system $(1000 \times)$ and these images were normalized to yield an average value of one inside and zero outside the aperture hole. Edge profiles perpendicular to the edge were extracted, averaged and further processed as described above. The curved edge of the aperture was found using the Plus operator (Verbeek \& van Vliet, 1994) with subpixel precision.

\subsection{Conversion factor and the detective quantum efficiency}

The conversion factor $C$ was measured by relating the beam current $I_{\text {beam }}$ and exposure time $t_{\exp }$ to the integrated intensity $O$ (in ADU) in the corrected output image. The beam diameter was made to be smaller than the field of view of the camera to ensure that the detector captured all incident electrons. The incident beam current was obtained through the Tecnai TEMscripting ActiveX $X$ server interface, which reads the current from the fluorescence screen. For all microscopes, the incident beam current readings were postcalibrated using independent current measurements from a Faraday cage of a double tilt analytical holder (Gatan, Inc., model 646). A Keithley model 602 was used as a picoamperemeter. The conversion factor $C$ in ADUs per primary electron $\left(\right.$ ADU pe ${ }^{-1}$ ) was calculated using the formula $C=\left(1.6 \times 10^{-19} O / I_{\text {beam }} t_{\exp }\right)$, where $t_{\exp }$ is the exposure time of the detector.

The MTF from the edge method was used for the DQE calculation. The NNPS was based on the subtraction of 
Table 1

Characteristics of three in-house 4k TEM detectors at $120 \mathrm{kV}$.

The single-chip sensors are read out from four different ports: upper left, lower left, upper right and lower right.

\begin{tabular}{|c|c|c|c|c|c|c|c|c|c|c|c|c|}
\hline \multirow[b]{2}{*}{ Quadrant } & \multicolumn{4}{|c|}{ Detector $X$} & \multicolumn{4}{|c|}{ Detector $Y$} & \multicolumn{4}{|c|}{ Detector $Z$} \\
\hline & $\begin{array}{l}\text { Upper } \\
\text { left }\end{array}$ & $\begin{array}{l}\text { Upper } \\
\text { right }\end{array}$ & $\begin{array}{l}\text { Lower } \\
\text { left }\end{array}$ & $\begin{array}{l}\text { Lower } \\
\text { right }\end{array}$ & $\begin{array}{l}\text { Upper } \\
\text { left }\end{array}$ & $\begin{array}{l}\text { Upper } \\
\text { right }\end{array}$ & $\begin{array}{l}\text { Lower } \\
\text { left }\end{array}$ & $\begin{array}{l}\text { Lower } \\
\text { right }\end{array}$ & $\begin{array}{l}\text { Upper } \\
\text { left }\end{array}$ & $\begin{array}{l}\text { Upper } \\
\text { right }\end{array}$ & $\begin{array}{l}\text { Lower } \\
\text { left }\end{array}$ & $\begin{array}{l}\text { Lower } \\
\text { right }\end{array}$ \\
\hline Bias (ADU) & 496 & 498 & 487 & 485 & 505 & 504 & 508 & 505 & 1003 & 1002 & 1002 & 1002 \\
\hline Readout noise (ADU) & 7.6 & 8.8 & 7.9 & 9.6 & 7.0 & 7.3 & 7.0 & 7.2 & 3.3 & 3.4 & 3.5 & 3.5 \\
\hline Readout noise (CCDe $\dagger)$ & 11.4 & 13.2 & 11.8 & 14.4 & 10.5 & 10.9 & 10.5 & 10.8 & 9.8 & 10.2 & 10.5 & 10.5 \\
\hline \multicolumn{13}{|c|}{ 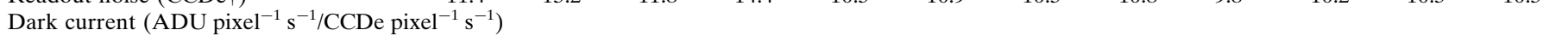 } \\
\hline Mean & $0.31 / 0.47$ & & & & $2.90 / 4.35$ & & & & $0.05 / 0.15$ & & & \\
\hline Standard deviation & $0.37 / 0.56$ & & & & $1.14 / 1.71$ & & & & $0.11 / 0.33$ & & & \\
\hline No. of pixels with $\overline{I_{\mathrm{dc}}}>50$ ADU pixel s${ }^{-1}$ & 144 & & & & 86 & & & & 9 & & & \\
\hline Effective gain (ADU pe ${ }^{-1}$ ) & 5.0 & 5.6 & 5.3 & 5.6 & 7.7 & 7.7 & 7.7 & 7.7 & 4.6 & 4.6 & 4.8 & 5.0 \\
\hline Conversion factor (ADU $\mathrm{pe}^{-1}$ ) & 76 & & & & 100 & & & & 34 & & & \\
\hline MTF at $0.5 \mathrm{Nq}(120 \mathrm{keV})$ & 0.12 & & & & 0.13 & & & & 0.19 & & & \\
\hline $\operatorname{DQE}(0)$ & 0.6 & & & & 0.6 & & & & 0.6 & & & \\
\hline DQE at $0.5 \mathrm{Nq}$ & 0.15 & & & & 0.16 & & & & 0.14 & & & \\
\hline
\end{tabular}

$\dagger$ The nominal gain was estimated to be $1.5 \mathrm{CCDe} / \mathrm{ADU}$ (binning 1) for detectors $X$ and $Y$ and 3 CCDe/ADU for detector $Z$.

two raw uniformly illuminated dark-subtracted images that were measured with the same exposure time $I_{\text {in }}=\left(I_{1}-I_{2}\right) / 2^{1 / 2} C$, with $I_{1}=I_{\text {white } 1}-\overline{I_{\mathrm{bg}}}$ and $I_{2}=I_{\text {white } 2}-\overline{I_{\mathrm{bg}}}$. The dose $N=\left\langle I_{1}+I_{2}\right\rangle_{x, y} / 2 C$ used for these images was 176,149 and 124 primary electrons per pixel for detectors $X, Y$ and $Z$, respectively. A sine-shaped windowing function $(w)$ was applied to this image in order to avoid edge artefacts from the implementation of the discrete Fourier transform (DFT). The square of the Fourier transform was multiplied by four to compensate for the power loss as a result of the windowing. Angular averaging of the spectrum was performed. The influence of the readout noise was represented by the term $\mathrm{NNPS}_{r}=\operatorname{DFT}^{2}\left[I_{\mathrm{rn}}(x, y) / C\right]$. The normalized noise power spectrum NNPS was obtained from (11) and (13) after dividing the contributions from the Poisson noise and readout noise by the dose $N$,

$$
\mathrm{NNPS}=\frac{\left\langle 4 \mathrm{DFT}^{2}\left(w I_{\mathrm{in}}\right)\right\rangle_{\varphi}+\mathrm{DFT}^{2}\left[\frac{I_{\mathrm{rn}}(x, y)}{C}\right]}{N} .
$$

After determining the NNPS, the DQE was computed using (12).

\section{Results}

\subsection{Outliers}

Fig. 1 presents the number of outliers versus integration time (for the dark reference images) or exposure time (for the white reference images) for each detector. Figs. 1(a) and 1(c) show a comparable increase of almost 1500 outliers in the dark reference images when increasing the integration time from milliseconds to $10 \mathrm{~s}$. This increase was not observed for detector $Y$ (Fig. 1b). The number of outliers in the white reference images was similar for all three detectors (Fig. 1d).

\subsection{Bias and the dark current}

The bias in the images can differ for each of the four quadrants, as each readout port has its own $\mathrm{AD}$ converter.
Fig. 2 shows a histogram of the bias for each quadrant of the three detectors. The average bias values for the four quadrants are 496, 498, 487 and 485 ADUs for detector $X, 505,504,508$ and 505 ADUs for detector $Y$, and 1003, 1002, 1002 and 1002 ADUs for detector $Z$ (Table 1).

Fig. 3 shows a histogram of the dark current for each of the three detectors. The average (standard deviation) of the dark current is $0.31(0.37), 2.9(1.14)$ and $0.05(0.11)$ ADU pixel ${ }^{-1} \mathrm{~s}^{-1}$ for detectors $X, Y$ and $Z$, respectively. Dark-current generation is a Poisson process. Therefore, it is to be expected that pixels with a high dark current will also have a high standard deviation of the dark current.

Pixels that have an excessive dark current are the so-called 'hot pixels'. A complementary cumulative distribution of these is shown in Fig. 4. The numbers of pixels with a dark current larger then 100, 50 and 30 ADU pixel ${ }^{-1} \mathrm{~s}^{-1}$ are 40, 144 and 675 for detector $X, 19,86$ and 853 for detector $Y$, and 4, 9 and 21 for detector $Z$, respectively.

\subsection{Readout noise}

Owing to the differences in readout circuitry, the readout noise is measured separately for each of the four quadrants of the image. The specification for the readout noise for a Fairchild CCD 486 Image Sensor is 8 ADU (12 e with 1.5 CCDe per ADU nominal gain). The mean of the readout noise is 8.5 ADU for detector $X, 7.1 \mathrm{ADU}$ for detector $Y$ and 3.4 ADU for detector $Z$ (Table 1). The nominal gain for each detector was determined from a comparison between full well capacity and saturation intensity in the image. It was estimated to be 1.5 CCDe per ADU for detectors $X$ and $Y$ and 3 CCDe per ADU for detector $Z$.

\subsection{Lookup tables}

Fig. 5(a) depicts the average outlier-corrected and background-corrected $I_{\text {gain }}$ image of detector $X$ with normalized gain values. In close-up (Fig. $5 b$ ), the image fibre bundles and even the individual fibres can be clearly seen. Fig. 5(c) shows a 
close-up of $I_{\text {gain }}$ of detector $Z$ displayed at the same magnification as in Fig. 5(b).

A mask is made for those pixels for which a very low signal was observed ( $I_{\text {gain }}<0.2$, e.g. owing to dust or broken fibres) or where the signal was excessively large $\left(I_{\text {gain }}>2\right.$, e.g. owing to thicker parts of the scintillator). The low and high threshold values ( 0.2 and 2.0, respectively) were selected empirically. Pixels within this mask could be either replaced by a value based on the mean and variance of the closest 'normal' neighbouring pixels or remain marked as 'unobserved' during subsequent processing. This mask forms a lookup table together with the list of pixel defects identified during the analysis of the dark reference images.

\subsection{Linearity of the response}

The linearity of the response was assessed by making a linear least-squares fit to the intensity of the white reference images versus exposure time. $R^{2}$ was calculated for every pixel. For all three detectors, the linear response was good within the range of intensity values measured: $R^{2}$ was higher than 0.999 for almost all pixels. It proved to be unnecessary to extend the mask of bad pixels (lookup table) with pixels that had a particular low $R^{2}$ value $($ e.g. $<0.9)$.

The average effective detector gain was calculated for each quadrant separately using both white reference and gradient images. Fig. 6 shows the effective gain for the upper right quadrant of the CCD using white reference images. Table 1 shows the effective gain in ADUs per primary electron for all quadrants. Detectors $X$ and $Z$ have a comparable effective gain of on average 5 ADU pe ${ }^{-1}$. Detector $Y$ gives more ADUs per primary electron (7.7) and its effective gain is very homogenous over each of the four quadrants. All three

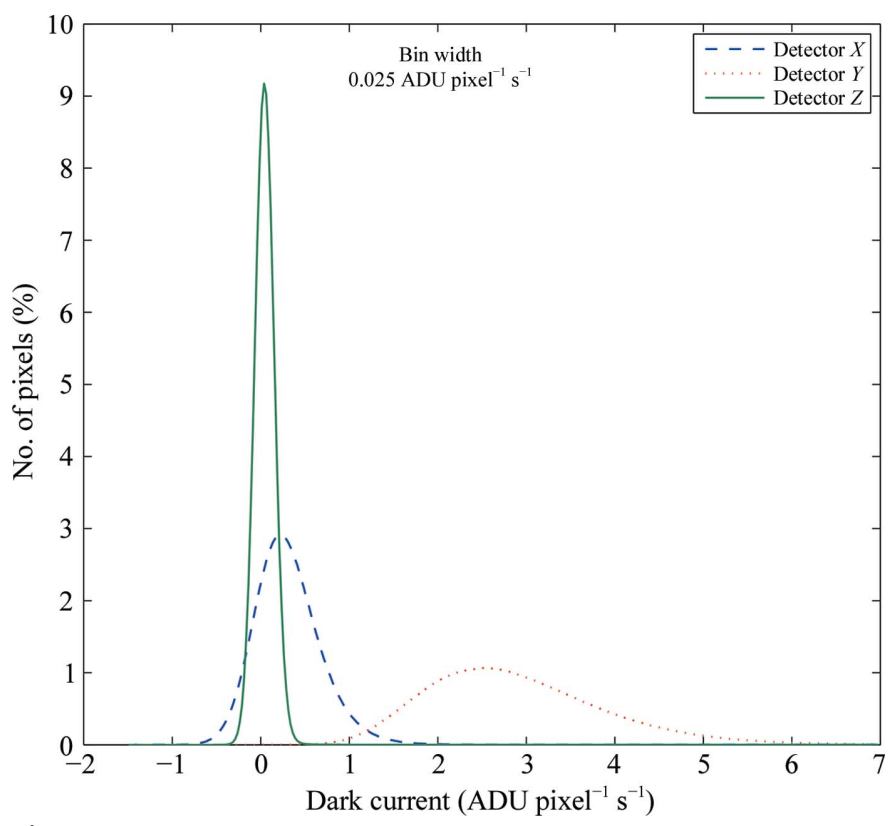

Figure 3

Histogram of the dark current for the three detectors. The bin width of the histogram is $0.025 \mathrm{ADU}$. Detector $Z$ has the smallest spread of the dark current. cameras showed excellent linearity of the variance in pixel response as a function of the pixel intensity. The method with gradient images was used for comparison and the effective gain values of the upper right quadrant were 5.6, 6.7 and 4.8 ADU pe ${ }^{-1}$ for detectors $X, Y$ and $Z$, respectively.

\subsection{MTF}

The modulation transfer function (MTF) was calculated with the noise (Fig. 7) and the edge methods (Fig. 8). Both the beam stop (Fig. $8 a$ ) and the diffraction aperture (Fig. 8b) were used to generate an edge. The MTF at half Nyquist was similar when determined using either of the two edge methods: 0.19 for detector $Z$ and 0.12 (beam-stop measurement) or 0.13 (aperture measurement) for detectors $X$ and $Y$. The MTF reached a higher minimum at higher frequencies for the noise method compared with the edge method.

The MTF of detector $Z$ was also determined at $200 \mathrm{kV}$ (Fig. 8a). It showed a more rapid decrease at lower frequencies. The MTF at half Nyquist was measured as 0.19 at $120 \mathrm{kV}$ and 0.13 at $200 \mathrm{kV}$.

\subsection{Conversion factor and DQE}

The conversion factors at $120 \mathrm{kV}$ as measured using the screen current method were 76, 100 and $34 \mathrm{ADU}_{\mathrm{pe}}^{-1}$ for detectors $X, Y$ and $Z$, respectively. The conversion factor for detector $Z$ at $200 \mathrm{kV}$ was 23 ADU pe ${ }^{-1}$. Fig. 9 shows the DQE for all three detectors. The DQE at frequencies close to zero is about 0.6 for all three detectors (Table 1 ).

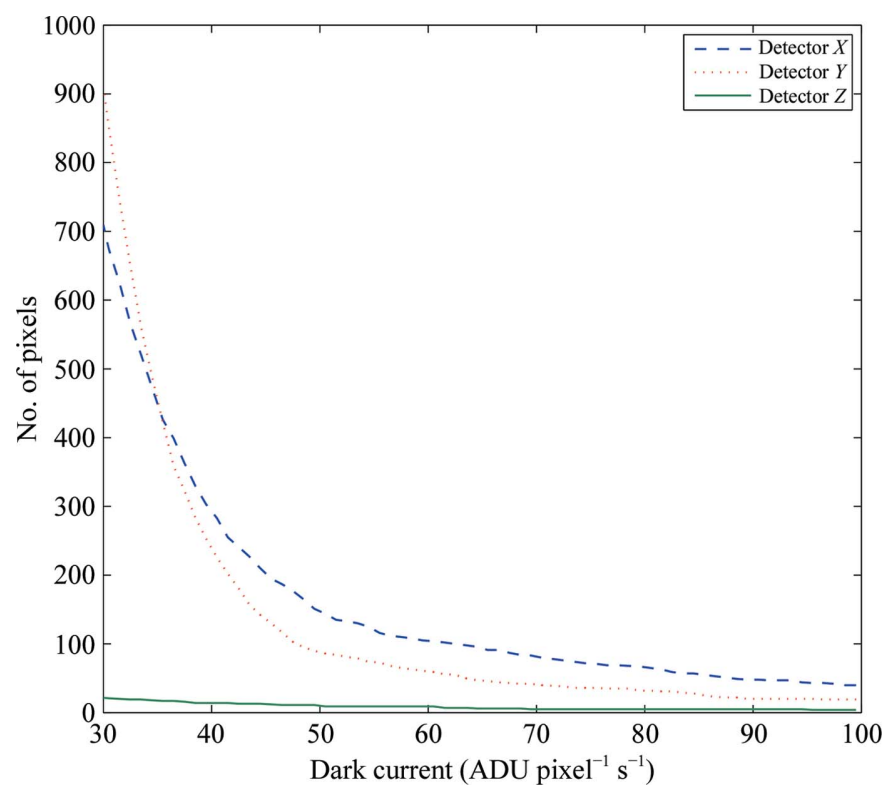

Figure 4

Complementary cumulative distribution function of the hot pixels, showing the number of pixels that have a dark current higher than a certain value. The number of pixels with a dark current larger then $100 \mathrm{ADU}$ pixel ${ }^{-1} \mathrm{~s}^{-1}$ is 40,19 and 4 for detectors $X, Y$ and $Z$, respectively. 


\section{Discussion}

Raw images provide useful system information. Quantification of noise based on raw (unprocessed) CCD images will give different values compared with quantification based on corrected (calibrated) images owing to image rescaling by flatfielding. In this study, the characterization of the cameras was based on raw images, which could fortunately be obtained through scripting for all of our in-house detectors. Data-

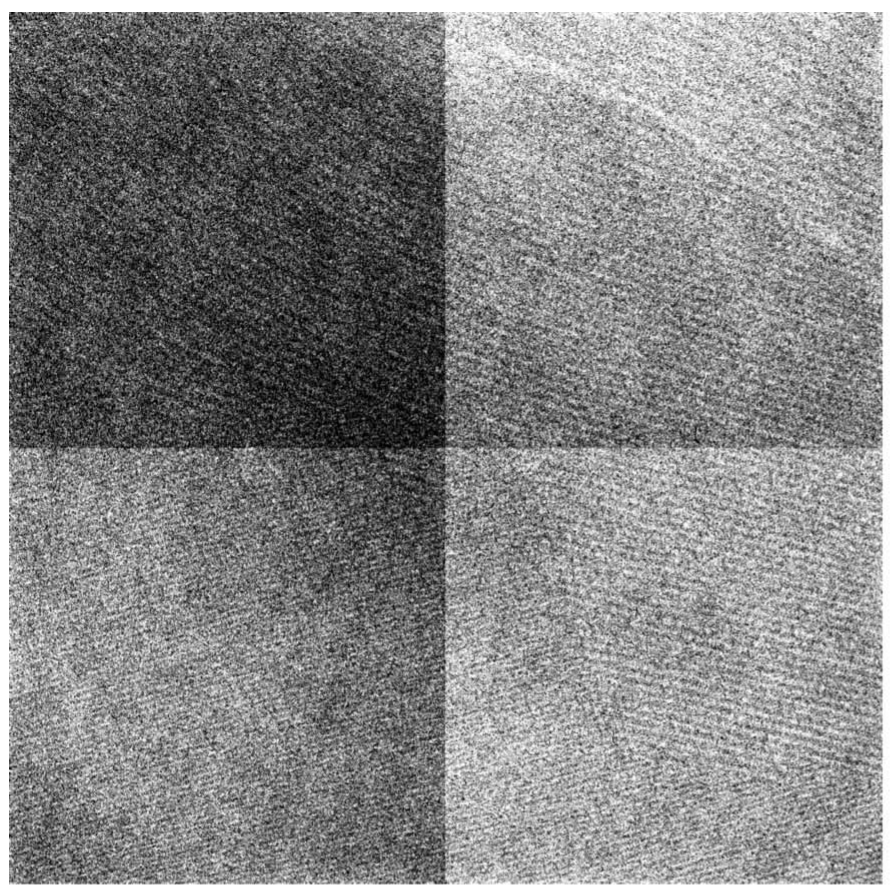

(a)

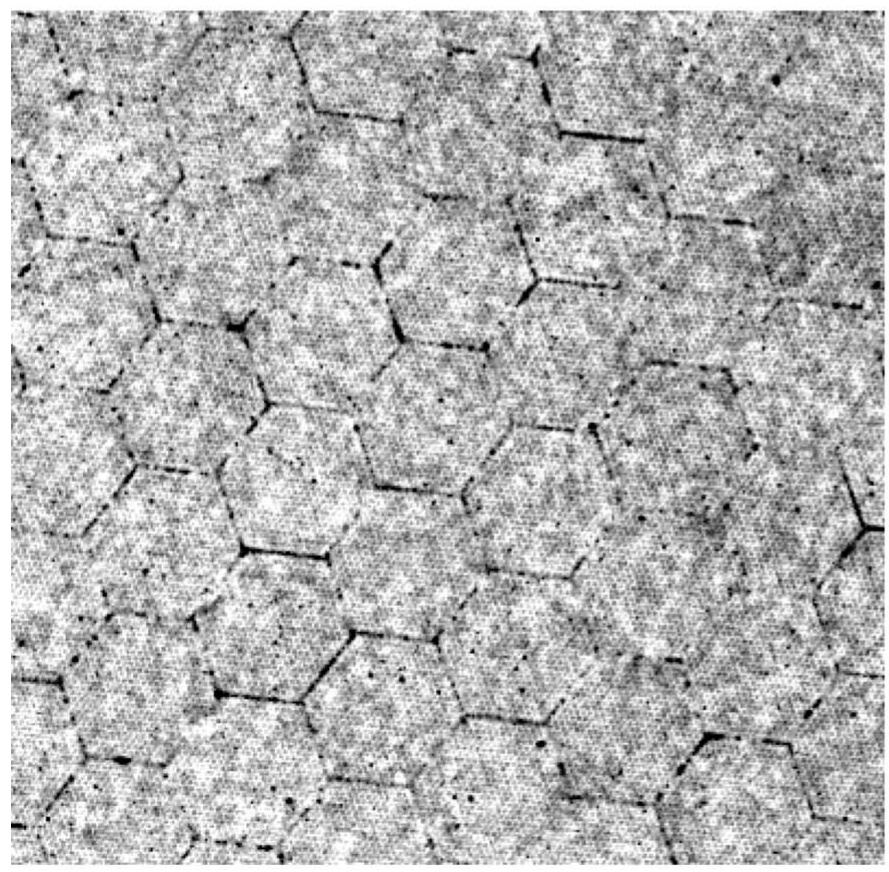

(b) acquisition software, such as Digital Micrograph (http:// www.gatan.com/products/software/) and Serial EM (Mastronarde, 2005), typically collect one new dark reference image prior to the collection of each new series of images, thus ensuring that the dark reference image noise was representative for the imaging conditions used. The disadvantage of this approach is that the acquisition of new dark reference images for every new series of images takes time. Multiple dark reference images would be needed in order to reject zingers. The FEI Tecnai software (v.3.1.2; http://www.fei.com) also allows online dark subtraction, but relies on previously collected dark reference images. These images are collected for one exposure time only, making dark subtraction less accurate if deviating exposure times were used. The possible advantage of the use of a series of previously collected dark reference images (apart from the gain in data-collection speed) is that more elaborate outlier-rejection schemes could be applied. Fig. 1 shows that the number of outliers, including decreased pixel values, can be quite substantial: up to one per 1000 (or $0.1 \%$ ) for detector $X$.

The number of outliers in the dark reference images increased for both detectors $X$ and $Z$ as a function of exposure time, at a rate of approximately 150 pixels s ${ }^{-1}$. This high rate can probably be attributed to the larger influence of cosmic rays and radioactive decay with increased integration time. In contrast, detector $Y$ did not show such an increase. This detector has a much higher dark current (Fig. 3; Table 1) compared with detectors $X$ and $Z$. The increased noise level of detector $Y$ at longer exposure times probably masks the detection of the increased occurrences of outliers as observed for the other two detectors. For all three detectors, the dark

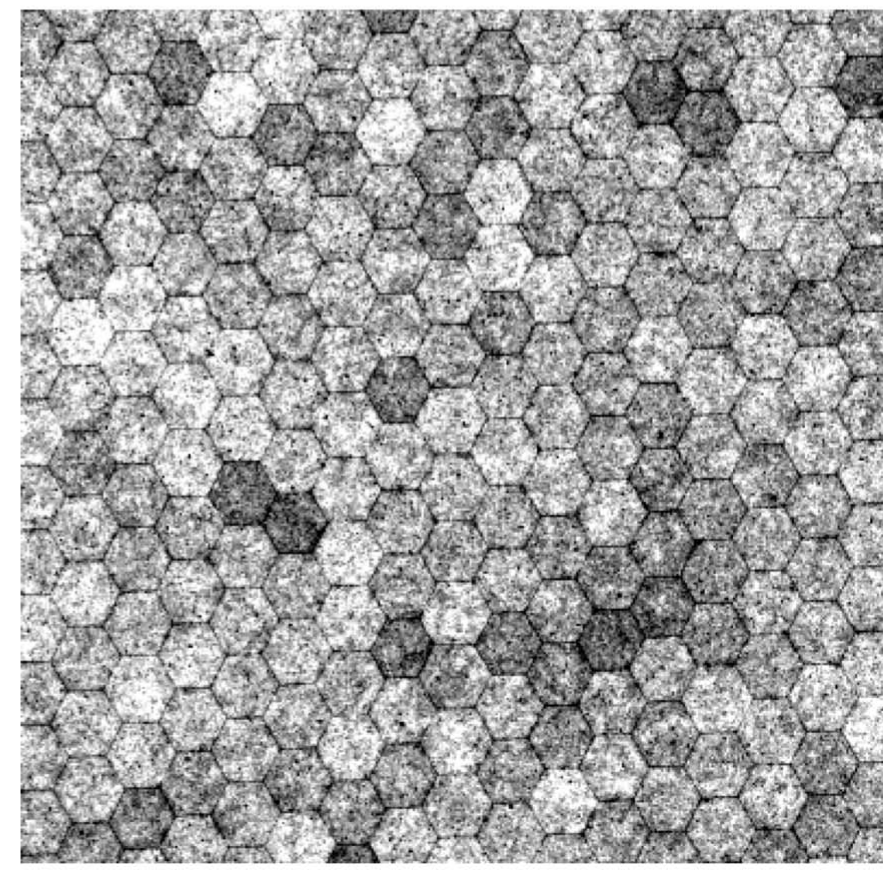

(c)

Figure 5

The dark-corrected and scaled $I_{\text {gain }}$ image. (a) Overview for detector $X$, showing the difference between the four quadrants. (b) Detail of $(a)$, showing the fibre-optic coupling and individual fibres. (c) Detail of $I_{\text {gain }}$ of detector $Z$, shown at the same magnification as in $(b)$. The size of the fibre bundles is about $1.1 \mathrm{~mm}$ for detectors $X$ and $Y$ and $450 \mu \mathrm{m}$ for detector $Z$. 
current is much higher then the tabulated nominal value of $0.005 \mathrm{CCDe}_{\text {pixel }}^{-1} \mathrm{~s}^{-1}$ for the 486 Fairchild sensor cooled to $213 \mathrm{~K}$. A doubling of the dark current for every $7 \mathrm{~K}$ of temperature increase (http://www.fairchildimaging.com/main/ documents/Condor486-90_RevE.pdf) would suggest that these sensors, despite their identical set temperature of $248 \mathrm{~K}$, are actually used at temperatures of $259 \mathrm{~K}$ (detector $X$ ), $281 \mathrm{~K}$ (detector $Y$ ) and $248 \mathrm{~K}$ (detector $Z$ ). An increased dark current could also be a consequence of radiation damage to the CCD itself (Allinson, 1994); however, detector $Y$ was basically new at the time of characterization. We interpret these values as a strong indication that detectors $X$ and $Y$ are not cooled as well as detector $Z$.

Macromolecular crystallography (MX) CCD sensors are generally cooled to far lower temperatures compared with TEM CCD sensors. For instance, the Bruker APEXII detector (based on the Fairchild 486 sensor) is cooled to $213 \mathrm{~K}$ and the

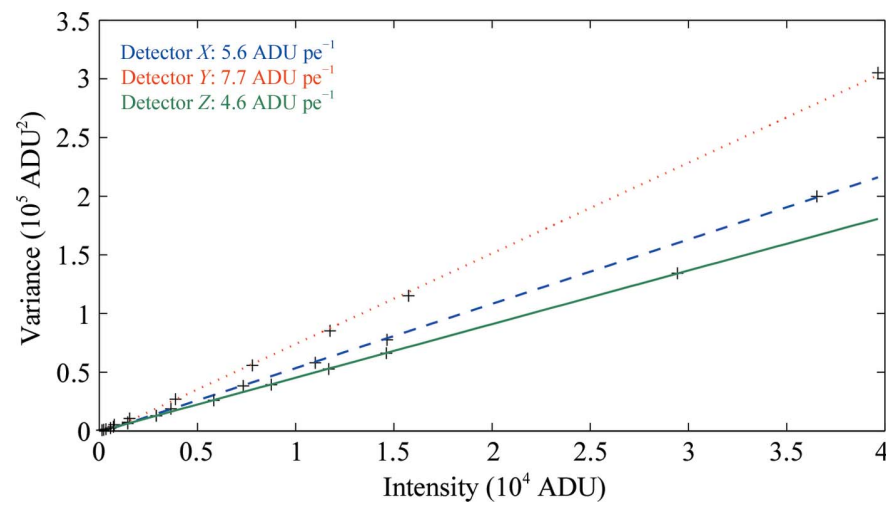

Figure 6

Effective gain measurements from white reference images of the upper right quadrant for detectors $X$ (dashed line), $Y$ (dotted line) and $Z$ (solid line). The values for the other quadrants are given in Table 1 .

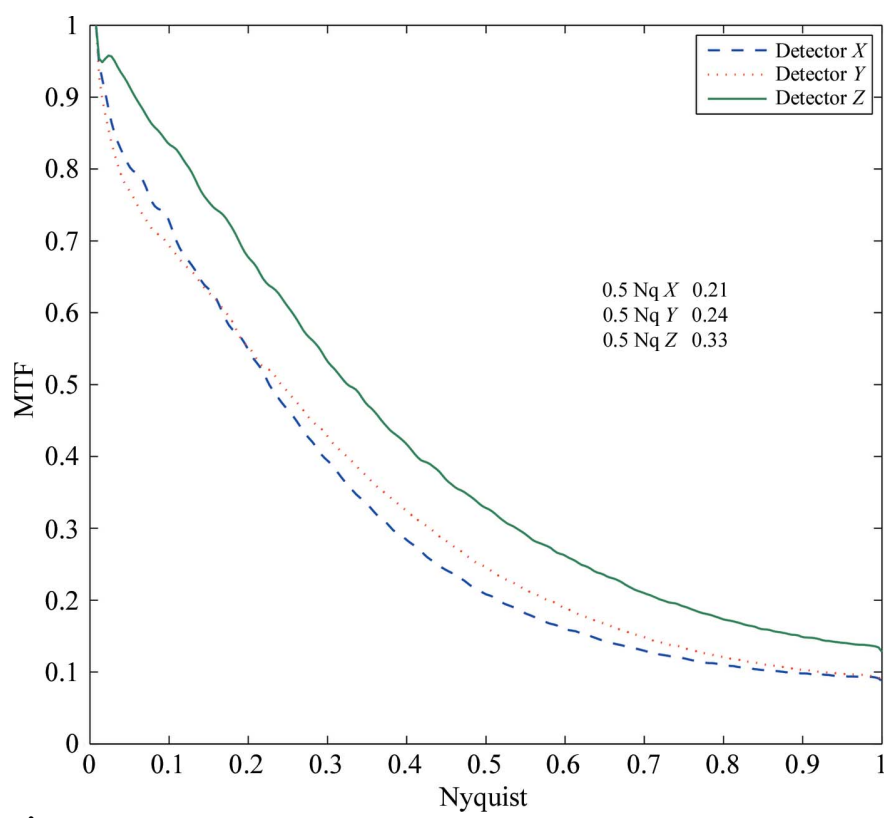

Figure 7

MTF obtained with the noise method. Radial averaging was performed with Gaussian rings in order to diminish the discretization error.
Rayonix 165 detector to $203 \mathrm{~K}$. The lower temperature is partly required because of the longer exposure times that are used at older X-ray sources and the lower conversion factors for X-ray photons compared with high-energy electrons. These X-ray detectors are thermally isolated units that are placed separately from the goniometer holding the specimen. This allows these detector manufacturers to accurately control the vacuum and temperature of the CCD, overcoming the need for routine recalibration. In contrast, TEM detectors are directly mounted on the electron microscope in a vacuum that is controlled by the electron-microscope manufacturer rather than the detector manufacturer. This vacuum also contains the specimen, films etc. and is therefore not guaranteed to be of

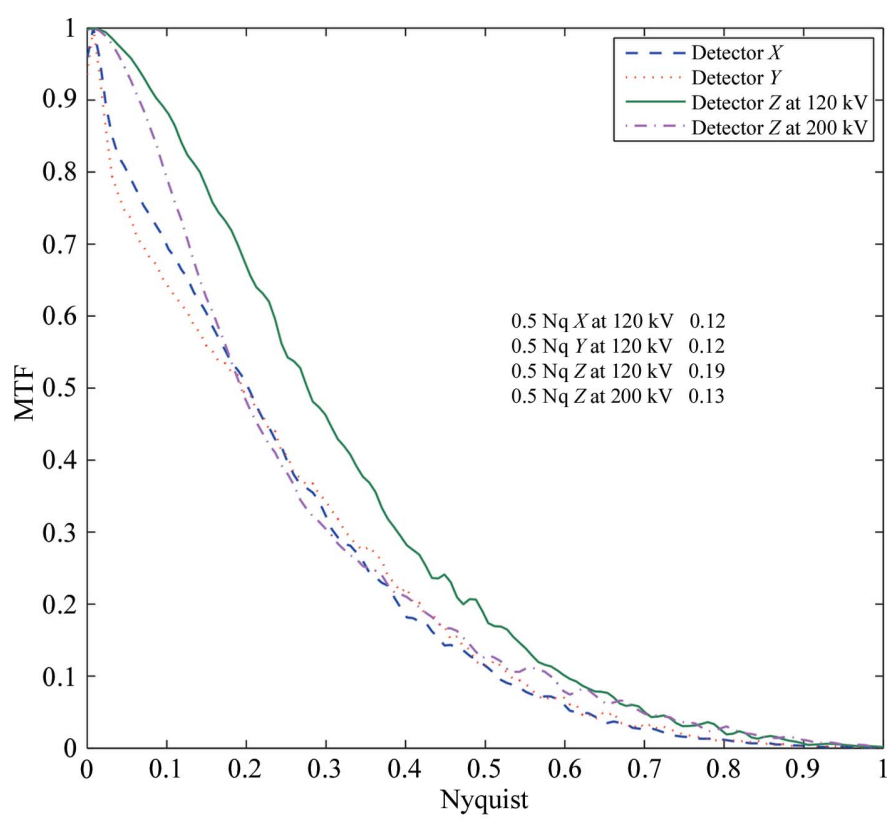

(a)

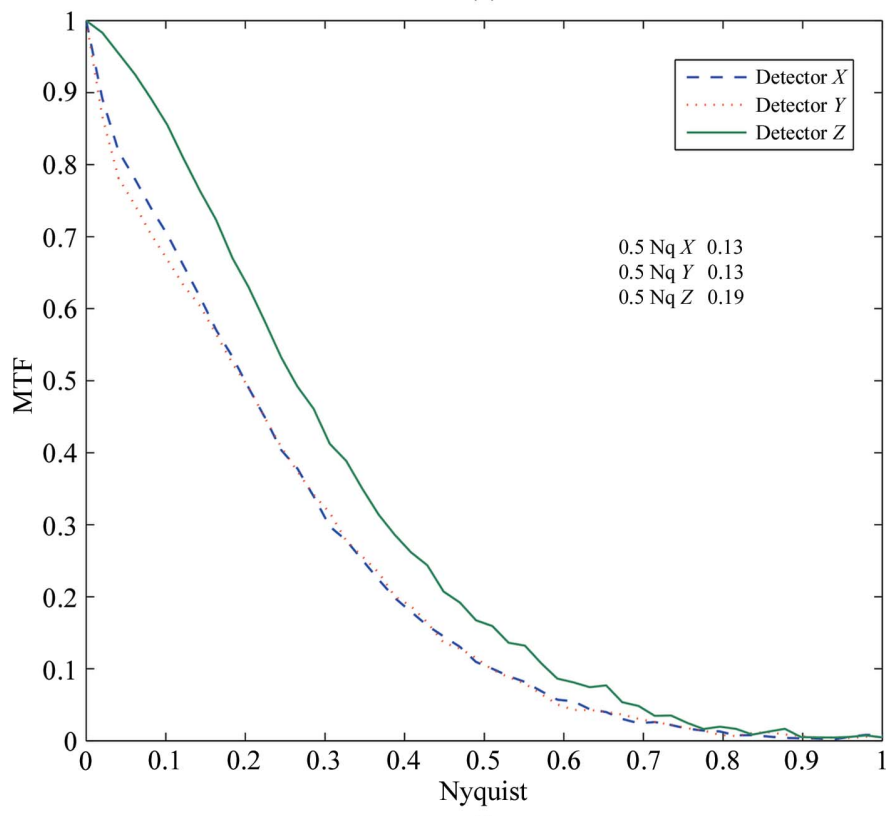

(b)

Figure 8

MTF obtained with the edge method employing $(a)$ a beam stop (detector $Z$ is characterized at 120 and $200 \mathrm{kV}$ ) and $(b)$ an aperture. 
constant quality. Deeper cooling of the CCD sensor and the coupled fibre-optic plate could result in condensation on the detector surface. In contrast to X-ray CCD detectors, TEM detectors do seem to require repetitive recalibrations. It is our impression that the frequency of calibration could be lessened if the vacuum and cooling conditions of the camera could be better controlled; i.e. to a standard comparable to those of MX detectors. A more constant and deeper cooling of the TEM detector would allow the use of more accurate bias-correction and gain-correction schemes, faster data collection (no need to recollect the dark image every time) and a decoupling of the correction for $\mathrm{CCD}$ fixed-pattern noise from the correction for beam inhomogeneities.

Fig. 4 shows the number of pixels with a dark current higher than a certain threshold. Various criteria can define a hot pixel, for example a dark current higher than ten times the average dark current or dark signals higher than one per 1000 of the maximum encoding range at the nominal exposure time (Ponchut, 2006). Fig. 4 seems to strongly favour detector $Z$ over detectors $X$ and $Y$, but this difference would be less striking if the first criterion had been used. The 'hottest' pixels, particularly for detector $X$, will saturate, with the column valves closed, if integration times between 10 and $60 \mathrm{~s}$ are used; leakage will result in pixel column defects. Not all detector manufacturers give image-blemish grades (point, cluster and column defects), as this is a delicate balance between system cost, industrial state of the art and actual experimental needs. As long as no recalibration of the detector is needed, 'hot' pixels can be reliably identified and taken into account during subsequent data processing by either replacing them with a value based on the statistics of neighbouring pixels or marking them as 'unknown'. This lookup table will also contain extreme values from the gainnormalized image as obtained using (5).

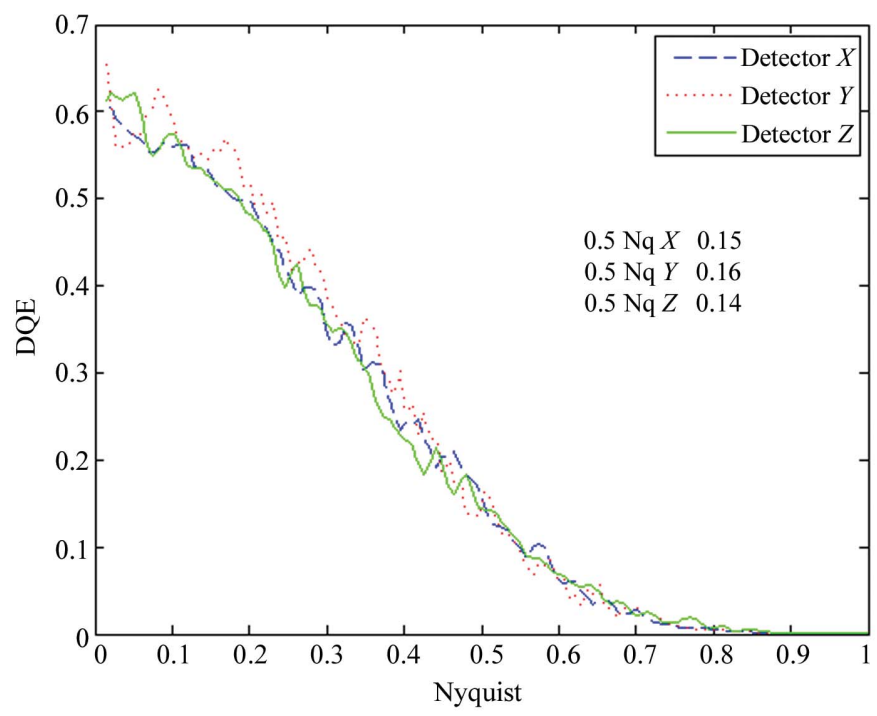

Figure 9

DQE for three detectors, measured with a dose of 182, 146 and 80 primary electrons per pixel for detectors $X, Y$ and $Z$, respectively. The similarity in the graphs indicates that the lower MTFs of detectors $X$ and $Y$ are compensated for by large conversion factors.
The impact of the use of lookup tables for image correction becomes particularly apparent during the calculation of crosscorrelation functions with the purpose of measuring image shifts. Image shifts are often measured in automated TEM procedures, e.g. during automated tomographic data collection (Koster et al., 1997). These automated procedures fail if image shifts are not measured correctly. Therefore, if the fixedpattern noise is not fully accounted for then images are not measured correctly because of the appearance of an additional undesired peak at the origin of the cross-correlation function. This origin peak corresponds to the unshifted fixed pattern between the two images. The height (intensity) of the origin peak can dominate the true cross-correlation peak when lowcontrast specimens such as vitrified biological materials are imaged. Under these conditions, the true correlation peak will be relatively low and the appearance of an origin peak owing to imperfect calibration may well pose limits to reliable automation. The use of lookup tables could mitigate part of the problem of fixed-pattern noise, but unfortunately not all software packages can employ these at present. Correction of the raw images with our own average dark and white reference images virtually eliminates the cross-correlation origin peak.

Uncorrected systematic outliers can result in undesirable artefacts if the data are used for three-dimensional reconstructions. State-of-the-art tomographic reconstruction packages such as IMOD (Mastronarde, 2008) and Inspect3D (http://www.fei.com) can use statistical criteria to identify and correct cosmic rays and detector flaws prior to reconstruction. However, more subtle systematic errors will still propagate unless adequate lookup tables are used.

The use of four readout ports of data from a CCD chip can result in both bias (Fig. 2) and gain (Fig. 5a) inhomogeneities. Gain inhomogeneities in corrected diffraction images of $\pm 1 \%$ or less with respect to the average values are deemed to be acceptable (Ponchut, 2006). The quadrant gain inhomogeneities in the raw images are less then $1 \%$ for detector $Z$, whereas they are around $6 \%$ for detectors $X$ and $Y$. An improper correction of poorly balanced offsets could lead to quadrant-edge effects, especially in Fourier domains (Zheng et al., 2007). Correction will be more precise if the spread of the bias is smaller.

The conversion factor is rather large for detectors $X$ $\left(76 \mathrm{ADU} \mathrm{pe} \mathrm{p}^{-1}\right.$ ) and $Y\left(100 \mathrm{ADU} \mathrm{pe}{ }^{-1}\right.$ ), whereas detector $Z$ has a conversion factor (34 ADU pe ${ }^{-1}$ at $120 \mathrm{kV}$, $23 \mathrm{ADU} \mathrm{pe}{ }^{-1}$ at $200 \mathrm{kV}$ ) that is close to the values given in the literature for this type of detector. The readout noise, in CCDe, is slightly higher for detector $X$ then for detectors $Y$ and $Z$ (Table 1). The effective gain is rather similar for detector $X \quad\left(5.3 \mathrm{ADU} \mathrm{pe}^{-1}\right)$ and for detector $Z$ (4.8 ADU pe ${ }^{-1}$ ), whereas detector $Y$ is the most sensitive, with an effective gain of $7.7 \mathrm{ADU} \mathrm{pe} \mathrm{p}^{-1}$. For simulated data, the effective gain will converge to the conversion factor for increased pixel binning as the dampening effect of the pointspread function will decrease for higher binning. For the data presented here, $16 \times 16$ rebinning reduces the difference between the effective gain and the conversion factor to less than $10 \%$. However, for higher binning the effective gain does 
not converge to the conversion factor owing to detectorresponse inhomogeneities (Ponchut, 2006).

Both the edge and the noise method give a comparable relative ranking of the three detectors. Detector $Z$ shows better propagation at low frequency compared with detectors $X$ and $Y$. Even for $200 \mathrm{kV}$ electrons, detector $Z$ looks better between 0 and 0.2 Nyquist rate compared with detectors $X$ and $Y$ for $120 \mathrm{kV}$ electrons, whereas it is comparable at higher frequencies. For higher voltages of the electron source, the percentage of electrons that are backscattered from the support layer of the CCD camera will be higher (Meyer \& Kirkland, 2000). They re-enter the scintillator and give rise to intensity at a large lateral distance from the place they initially hit the scintillator and cause a more rapid decrease of the signal for low frequencies. By changing the thickness of the phosphor layer one can alter the balance between sensitivity and resolution, as a thicker layer gives a better sensitivity but also a larger point spread. This might explain why detectors $X$ and $Y$ have better sensitivity but lower resolution compared with detector $Z$, although the differences in the size of the fibre-optic bundles (1.1 mm for detector $X$, Fig. $5 b ; 450 \mu \mathrm{m}$ for detector $Z$, Fig. $5 c$ ) are also likely to have an effect on the MTF at low resolution. The noise method gives values for the MTF that are too optimistic at higher frequencies where the noise contributions of the camera start to dominate. Both the beam-stop (Fig. 8b) and the aperture (Fig. 8a) MTF graphs approximate zero towards the Nyquist frequency, which has been reported to be an over-pessimistic estimate of the true MTF (Fan \& Ellisman, 2000).

For all three detectors, the DQE at frequencies close to zero is about 0.6. Measurement errors in conversion factor would give proportional errors in the DQE measurement (10). TEM detectors with larger pixel sizes can show even better $\mathrm{DQE}(0)$ values of 0.8 (Kim et al., 2007) or 0.76 (Meyer et al., 2000). The normalized noise power spectrum will be dose-dependent; Fig. 9 shows the DQE for our three detectors measured with a relatively high dose of 182,146 and 80 primary electrons per pixel. Overall, the DQEs of the more sensitive detectors $X$ and $Y$ are remarkably comparable with the DQE of the sharper and less noisy detector $Z$ (Fig. 9).

A number of programs exist to aid macromolecular crystallographers in planning their data-collection strategy (Leslie, 1992; Ravelli et al., 1997; Popov \& Bourenkov, 2003). From one or a few images, these programs will characterize the specimen, simulate data-set statistics for different combinations of data-collection parameters and suggest the most optimal ones. The program Best (Popov \& Bourenkov, 2003) honours its name by being able to suggest an optimal datacollection strategy based on the most complete set of parameters. These include anisotropic diffraction, background scattering, detector statistics, geometric parameters and even radiation damage (Bourenkov \& Popov, 2006; Ravelli \& Garman, 2006). Given a number of test images, the program will suggest exposure time, rotation range, number of images and starting angle and predict data-set statistics such as signalto-noise versus resolution for each suggestion. One could imagine a similar scheme for (cryo-)electron microscopy, in particular for tomographic data collection, where the effect of parameters such as defocus, rotation steps, number of angles, single versus double tilt, electron dose and electron-dose rate, magnification and detector binning could be simulated after an initial characterization of the specimen with a small number of test images. A detailed knowledge of all the parameters involved, including the characteristics of the camera as determined here, will aid the development of an 'expert system' (Leslie et al., 2002) that will help the electron microscopist to make objective and reproducible decisions for their (tomographic) data collection.

\section{Conclusion}

A general methodology for characterizing TEM CCD detectors has been presented. The set of algorithms have been added to the publicly available image-processing toolbox for $M A T L A B$ (http://www.diplib.org/home22266) to allow nonexpert electron-microscopy users to characterize, based on uncorrected images, the properties of their CCD detector. Furthermore, it can facilitate information exchange between detector users and producers. Three $4 \mathrm{k}$ in-house CCDs have been characterized, showing different strengths in terms of sensitivity, resolution, DQE and noise. The need for the use of lookup tables is demonstrated. Fixed-pattern noise could be fully accounted for by using large sets of dark and white reference images. Unfortunately, the noise patterns seem to drift in time, possibly because of unstable cooling of the CCD sensors, thereby limiting the useful lifetime of these reference sets.

The authors thank Frank Faas and Montserat Barcena for useful discussions, Remco Schoenmakers for critical reading of the manuscript and Paul Mooney for crucial help in the determination of the conversion factors by calibration of the incident beam currents. MV was financially supported by the FOM Industrial Partnership program No. 07.0599. RBGR acknowledges financial support from NWO under project No. 016.072.321. This article includes work presented at The Max-Inf2/Lorentz Center Workshop on New Algorithms in Macromolecular Crystallography and Electron Microscopy, May 2008.

\section{References}

Aikens, R. S., Agard, D. A. \& Sedat, J. W. (1989). Methods Cell Biol. 29, 291-313.

Allinson, N. M. (1994). J. Synchrotron Rad. 1, 54-62.

Bourenkov, G. P. \& Popov, A. N. (2006). Acta Cryst. D62, 58-64.

Bourgeois, D., Moy, J. P., Svensson, S. O. \& Kvick, A. (1994). J. Appl. Cryst. 27, 868-877.

Brink, J. \& Chiu, W. (1994). J. Struct. Biol. 113, 23-34.

Carragher, B., Kisseberth, N., Kriegman, D., Milligan, R. A., Potter, C. S., Pulokas, J. \& Reilein, A. (2000). J. Struct. Biol. 132, 33-45.

Dainty, J. C. \& Shaw, R. (1974). Image Science: Principles, Analysis and Evaluation of Photographic-type Imaging Processes. New York: Academic Press.

Downing, K. H. \& Hendrickson, F. M. (1999). Ultramicroscopy, 75, 215-233. 
Fan, G. Y. \& Ellisman, M. H. (2000). J. Microsc. 200, 1-13.

Faruqi, A. R., Cattermole, D. M., Henderson, R., Mikulec, B. \& Raeburn, C. (2003). Ultramicroscopy, 94, 263-276.

Faruqi, A. R. \& Henderson, R. (2007). Curr. Opin. Struct. Biol. 17, 549-555.

Kim, Y. M., Lee, J. Y., Moonen, D., Jang, K. I. \& Kim, Y. J. (2007). J. Electron Microsc. 56, 217-224.

Koster, A. J., Chen, H., Sedat, J. W. \& Agard, D. A. (1992). Ultramicroscopy, 46, 207-227.

Koster, A. J., Grimm, R., Typke, D., Hegerl, R., Stoschek, A., Walz, J. \& Baumeister, W. (1997). J. Struct. Biol. 120, 276-308.

Krivanek, O. L. \& Mooney, P. E. (1993). Ultramicroscopy, 49, 95-108.

Leslie, A. G. W. (1992). Jnt CCP4/ESF-EACBM Newsl. Protein Crystallogr. 26.

Leslie, A. G. W., Powell, H. R., Winter, G., Svensson, O., Spruce, D., McSweeney, S., Love, D., Kinder, S., Duke, E. \& Nave, C. (2002). Acta Cryst. D58, 1924-1928.

Mastronarde, D. N. (2005). J. Struct. Biol. 152, 36-51.

Mastronarde, D. N. (2008). J. Microsc. 230, 212-217.

McMullan, G., Cattermole, D. M., Chen, S., Henderson, R., Llopart, X., Summerfield, C., Tlustos, L. \& Faruqi, A. R. (2007). Ultramicroscopy, 107, 401-413.

Meyer, R. R. \& Kirkland, A. I. (2000). Microsc. Res. Tech. 49, 269-280.

Meyer, R. R., Kirkland, A. I., Dunin-Borkowski, R. E. \& Hutchison, J. L. (2000). Ultramicroscopy, 85, 9-13.

Moy, J. P., Hammersley, A. P., Svensson, S. O., Thompson, A., Brown, K., Claustre, L., Gonzalez, A. \& McSweeney, S. (1996). J. Synchrotron Rad. 3, 1-5.

Mullikin, J. C., van Vliet, L. J., Netten, H., Boddeke, F. R., van der Feltz, G. W. \& Young, I. T. (1994). Proc. SPIE, 2173, 73-84.
Nickell, S., Forster, F., Linaroudis, A., Net, W. D., Beck, F., Hegerl, R., Baumeister, W. \& Plitzko, J. M. (2005). J. Struct. Biol. 149, 227234.

Ponchut, C. (2006). J. Synchrotron Rad. 13, 195-203.

Popov, A. N. \& Bourenkov, G. P. (2003). Acta Cryst. D59, 11451153.

Rabbani, M., Shaw, R. \& Van Metter, R. (1987). J. Opt. Soc. Am. A, 4, 895-901.

Ravelli, R. B. G. \& Garman, E. F. (2006). Curr. Opin. Struct. Biol. 16, 624-629.

Ravelli, R. B. G., Sweet, R. M., Skinner, J. M., Duisenberg, A. J. M. \& Kroon, J. (1997). J. Appl. Cryst. 30, 551-554.

Roberts, P. T. E., Chapman, J. N. \& MacLeod, A. M. (1982). Ultramicroscopy, 8, 385-396.

Samei, E., Flynn, M. J. \& Reimann, D. A. (1998). Med. Phys. 25, 102-113.

Sander, B., Golas, M. M. \& Stark, H. (2005). J. Struct. Biol. 151, 92-105.

Spence, J. C. H. \& Zuo, J. M. (1988). Rev. Sci. Instrum. 59, 21022105.

Suloway, C., Pulokas, J., Fellmann, D., Cheng, A., Guerra, F., Quispe, J., Stagg, S., Potter, C. S. \& Carragher, B. (2005). J. Struct. Biol. 151, 41-60.

Verbeek, P. W. \& van Vliet, L. J. (1994). IEEE Trans. Pattern Anal. Mach. Intell. 16, 726-733.

Vliet, L. J. van, Sudar, D. \& Young, I. T. (1998). Cell Biology, edited by J. E. Celis, pp. 109-120. New York: Academic Press.

Zheng, S. Q., Keszthelyi, B., Branlund, E., Lyle, J. M., Braunfeld, M. B., Sedat, J. W. \& Agard, D. A. (2007). J. Struct. Biol. 157, 138-147.

Zinger, A. (1961). Biometrika, 48, 457-461. 